Original Article

\title{
Correlation between radiographic sagittal alignment, range of motion, muscle strength, and quality of life in adults with spinal deformities
}

\author{
Yoshiki Saimon, RPT, JSPO-AT, MSc ${ }^{1,2)^{*}}$, Ah-Cheng Goh, PT, $\mathrm{PhD}^{2)}$, \\ Kimito Momose, RPT, PhD ${ }^{1)}$, Daichi Ryuzaki, RPT ${ }^{3)}$, Hiroyasu AKahane, RPT, JSPO-AT ${ }^{1,3)}$, \\ Akemi Oba, RPT ${ }^{3)}$, Keijiro Mukaiyama, MD, $\mathrm{PhD}^{4)}$ \\ 1) Graduate School of Medicine, Shinshu University, Japan \\ 2) Faculty of Health Sciences, Iryo Sosei University: 5-5-1 Chuodai Iino, Iwaki-shi, \\ Fukushima 970-8551, Japan \\ 3) Department of Rehabilitation, North Alps Medical Center Azumi Hospital, Japan \\ 4) Department of Orthopedic Surgery, North Alps Medical Center Azumi Hospital, Japan
}

\begin{abstract}
Purpose] The aims of this study were to examine the relationship between range of motion/muscle strength of the spine and lower limbs with 1) radiographic sagittal alignment and 2) quality of life of participants with spinal deformities to adequately target the appropriate factors for effective treatment. [Participants and Methods] This study used an observational cross-sectional correlational design. Participants with spinal deformities and low back pain were recruited for the study. The dependent variables were range of motion/muscle strength of the spine and lower limbs, sagittal alignment, and quality of life. [Results] Regarding alignment, significant correlations were found between hip extension range of motion and sagittal vertebral axis; between occiput-to-wall distance and thoracolumbar kyphosis; and between back extensor endurance and pelvic tilt and pelvic incidencelumbar lordosis mismatch, also known as the calculated (pelvic incidence) minus (lumbar lordosis) value. With regards to quality of life, significant correlations were found between pain-related disorders and lumbar spine range of motion, and between gait disturbance and knee and hip extension ranges of motion. [Conclusion] Our findings suggest that these factors should be targeted for assessment and treatment of adults with spinal deformities. Key words: Sagittal alignment, Range of motion, Muscle strength
\end{abstract}

(This article was submitted Oct. 4, 2019, and was accepted Nov. 20, 2019)

\section{INTRODUCTION}

In adults with spinal deformities, one of the factors leading to a decreased in quality of life (QOL) is deformities in sagittal plane alignment (kyphosis), rather than coronal plane alignment (scoliosis) ${ }^{1}$. Kyphosis of the spinal column has been reported to increase with age in healthy individuals ${ }^{2-4}$. In these studies, kyphosis due to natural age-related changes in the spine or due to reduced activity did not significantly affect the patient's QOL. However, with the increased in life expectancy in most developed countries including Japan, coupled with the increase in activity in elderly people, spinal deformities associated with pathological conditions have gained renewed attention and it is predicted that the number of patients requiring medical attention related to their spinal deformities will increase in the future ${ }^{5)}$.

To evaluate sagittal alignment, kyphometers ${ }^{6,7)}$, inclinometers ${ }^{8)}$, flexicurves ${ }^{6,8)}$, Spinal Mouse ${ }^{9)}$ and other methods are usually performed. In orthopedics, the gold standard for evaluation of sagittal alignment is considered to be standing lateral spine radiographs ${ }^{10)}$. From this, sagittal alignment of the spine and pelvis, as well as sagittal global alignment, are measured

*Corresponding author. Yoshiki Saimon (E-mail: yoshiki.saimon@isu.ac.jp)

(C2020 The Society of Physical Therapy Science. Published by IPEC Inc.

(c) (1) $\odot$ This is an open-access article distributed under the terms of the Creative Commons Attribution Non-Commercial No DerivacC. 
as X-ray parameters (Fig. 1). In a study examining the relationship between sagittal alignment and QOL for adults with spinal deformities using standing lateral spine radiographs, Glassman et al. ${ }^{11)}$ reported that the severity of symptoms increased and QOL decreased as the spinal kyphosis increased (particularly in the lumbar region) and the anterior deviation of the C7 plumb line from S1 spinal segment increased (referred to as positive sagittal imbalance). Furthermore, Yamato et al. ${ }^{1)}$ reported that in Japanese adults with spinal deformities, increased pelvic retroversion angle was correlated with QOL decline, in addition to the deterioration of the global sagittal alignment and lumbar kyphosis.

Although pathology in the sagittal alignment of the spinal column is complex, associations with other physical characteristics have been reported. Spinal extensor muscle strength has been negatively correlated with thoracic kyphosis, and positively correlated with increased lumbar lordosis and pelvic anteversion ${ }^{12)}$. Balzini et al. ${ }^{13)}$ reported that participants with severe sagittal alignment resulting in flexed postures also had severe muscle impairment such as weaker spinal extensors, ankle plantarflexors and dorsiflexors, and shorter muscle length in the pectoralis and hip flexor muscles. Kai et al. ${ }^{14)}$ also reported a correlation between decreased lumbar lordosis and decreased quadriceps muscle strength. In addition, an increase in thoracic kyphosis and postural stiffness has been shown to be associated with increased aging and other pathological conditions $^{3)}$. Moal et al. ${ }^{15}$ ), in their study on adults with spinal deformity suggested that muscular degeneration occurred most commonly in spinal and hip extensor muscles. Therefore, these studies suggest that there could also be a relationship between sagittal alignment of the spine and spinal range of motion (ROM) and muscle strength, and also with the lower limb ROM and muscle strength. In a systematic review of exercise therapy for patients with kyphosis ${ }^{16}$, a small number of randomized controlled trials indicated a slight improvement in sagittal alignment by improving spinal extensor strength. However, they were unable to conclusively determine the effect of exercise therapy on sagittal alignment in kyphosis patients due to the poor homogeneity in and quality of the RCTs ${ }^{16)}$. In addition, the RCTs cited in this systematic review ${ }^{16)}$ did not standardize the site and purpose of exercise therapy as it relates to physical function.

There are reports showing the relationship between sagittal alignment and the ROM/muscle strength of the spine and lower limbs ${ }^{3,12-14)}$; and between sagittal alignment and $\mathrm{QOL}^{1,11)}$ in adults with spinal deformities. However, there have been no studies investigating the relationship between sagittal alignment and QOL with ROM/muscle strength of the spine and lower extremities in adults with spinal deformities. In addition, most of these studies did not adopt the gold standard for measuring sagittal alignment, i.e. radiographic imaging, and therefore, the validity of their results may be questionable.

Therefore, the objectives of this study are to examine the relationship between ROM as well as muscle strength of the spine and lower limbs with 1) radiographic sagittal alignment and 2) QOL. We believe that an investigation into the relationship among ROM, muscle strength, sagittal alignment and QOL is necessary so that the target of exercise therapy can be appropriately determined and more effective exercise therapy can be performed.
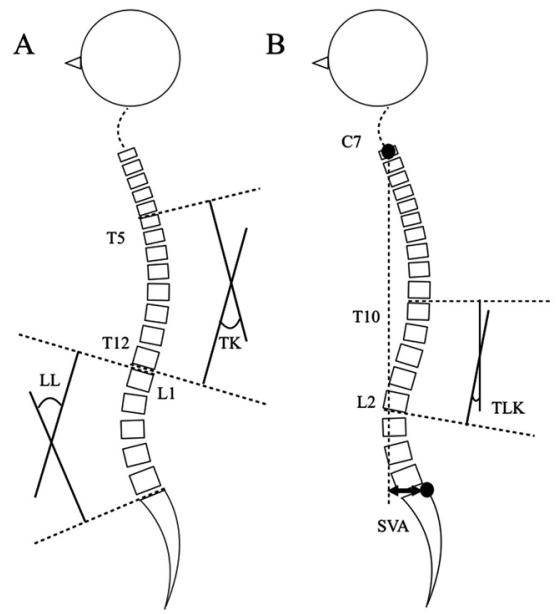

C

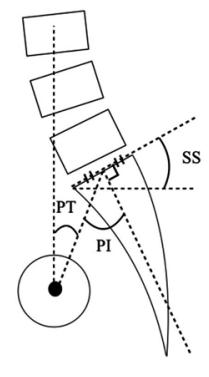

Fig. 1. Parameters for sagittal alignment. (A) Thoracic kyphosis (TK) was measured as the angle between the upper endplate of the T5 and the lower endplate of the T12 vertebral bodies. Lumbar lordosis (LL) was measured as the angle between the upper endplate of the L1 and the upper endplate of the S1 vertebral bodies. (B) Thoracolumbar kyphosis (TLK) was measured as the angle between the upper endplate of the T10 and the lower endplate of the L2 vertebral bodies. Sagittal vertical axis (SVA) was measured as the distance between the $\mathrm{C} 7$ plumb line and the posterior upper endplate of S1. (C) Pelvic incidence (PI) was measured from the midpoint of the upper endplate of S1, the angle between the line perpendicular to the midpoint and the line to the center of the femoral head. Pelvic tilt (PT) was measured from the midpoint of the upper endplate of S1, the angle between the line to the center of the femoral head and the vertical line. Sacral slope (SS) was measured as the angle between the upper endplate of the $\mathrm{S} 1$ and the horizontal line. 


\section{PARTICIPANTS AND METHODS}

This study employed an observational cross-sectional correlational study design. Ethical approval was obtained from the Graduate School of Medicine, Shinshu University (Approval No. 3993) and North Alps Medical Center Azumi Hospital (Approval No. 28-10-2). Participants were recruited from the latter institution from December 2016 to March 2019.

A visiting Orthopedic surgeon referred patients with spinal deformities and who had low back pain to the study. At the initial visit to the physical therapy department, the outline of the study was explained and written informed consent obtained. Participants were excluded if they had lower limb pain, neurological disorders, recent spinal compression fractures or ankylosis, and hip disorders. A total of 29 volunteers were recruited for this study. However, three of them failed to meet the inclusion criteria, and the remaining 26 participated in the study $(10$ males and 16 females, mean \pm standard deviation: age, $78.6 \pm 7.5$ years; height, $158.0 \pm 7.7 \mathrm{~cm}$; body weight, $57.3 \pm 10.2 \mathrm{~kg}$ ). From the 26 participants, there was some dropout for some of parameters measured ranging from 1 to 17 . This will be highlighted further in the results and discussion sections.

Prior to commencement, a pilot study was carried out to assess the reliability of measurement procedures used in this study ${ }^{17)}$. The results demonstrated a high inter-rater reliability with $\mathrm{ICC}_{1,1}$ for $\mathrm{ROM}$ ranging from 0.89 to 0.98 , and muscle strength ranging from 0.86 to 0.98 .

Regarding measurement of the spine and pelvis alignment, lateral spine radiographs were taken in standing. In this position, the upper limb was placed in the clavicle position as described by Horton et al ${ }^{18)}$. The X-ray parameters were measured from the lateral view by an orthopedic surgeon. The X-ray parameters measured (Fig. 1) included the following: (1) sagittal spinal alignment: (a) thoracic kyphosis (TK) - the angle between the upper endplate of the T5 and the lower endplate of the T12 vertebral bodies; (b) thoracolumbar kyphosis (TLK) - the angle between the upper endplate of the T10 and the lower endplate of the L2 vertebral bodies; (c) lumbar lordosis (LL) - the angle between the upper endplate of the L1 and the upper endplate of the S1 vertebral bodies); (2) global sagittal alignment: (a) sagittal vertical axis (SVA) - distance between the C7 plumb line and the posterior upper endplate of S1; (3) sagittal pelvic alignment: (a) pelvic incidence (PI) - from the midpoint of the upper endplate of S1, the angle between the line perpendicular to the midpoint and the line to the center of the femoral head; (b) pelvic tilt (PT) - from the midpoint of the upper endplate of S1, the angle between the line to the center of the femoral head and the vertical line; (c) sacral slope (SS) - the angle between the upper endplate of the S1 and the horizontal line.

To fully understand the spinal alignment parameters, it is necessary to consider the pelvic incidence (PI, a morphological parameter), the sacral slope and the sagittal curve (LL, lumbar lordosis) ${ }^{19}$ ). Schwab et al. ${ }^{20)}$ suggested that the pelvic incidence minus lumbar lordosis mismatch, which is a calculated value (PI - LL) of an angle greater than 10 degrees is an indication of the severity for adult spinal deformity.

Regarding measurement of ROM, the parameters measured included (1) spinal ROM and (2) lower limb ROM. The parameters related to (1) spinal ROM are: (a) modified occiput to wall distance (OWD): The participant stood against the wall, with the heel and back touching the wall, and the lower limbs/vertebral column actively extended as much as possible. With the cervical spine in neutral position, the participant was instructed to push the head towards the wall as much as possible, and the distance between the wall and the occiput was measured; (b) prone press-up test (PPUT): From the prone position, the distance between the sternal notch and the bed was measured with the trunk passively extended as much as possible using both upper limbs. Participants were instructed not to lift the pelvis off the bed, with the examiner confirming that the bilateral anterior superior iliac spine were always in contact with the bed ${ }^{21)}$; (c) from the lateral X-ray image in side lying, the total ROM between the maximum flexion and maximum extension positions were measured by the lines from the upper endplates of the L1 and S1. Measurements were performed by the same examiner twice, and each measurement was performed at intervals of one day or more. The average of two measurements was recorded; (d) a smartphone (iPhone 5, Apple Inc., Cupertino, CA, USA) with an inclinometer app (TiltMeter-advanced level, downloaded from App Store) was used $^{22}$. The measurement was performed between the spinous processes of C7/T1 and T12/L1. For the maximum flexion position, participants were instructed to bend forward as much as possible while sitting on a bed. For the maximum extension position, participants were instructed to lift their upper trunk off the bed from the prone position. Each measurement was performed twice by the same examiner, and the average value was recorded.

For the parameters related to (2) lower limb ROM, all measurements were carried out using a goniometer by two examiners with one examiner performing the passive movement, and the other measuring the ROM as follows: (a) hip flexion and knee flexion/extension ROM were measured according to the Japanese Orthopedic Association and The Japanese Association of Rehabilitation Medicine; (b) hip extension ROM was measured in side lying position with the tested leg uppermost, and the non-tested leg in slight hip flexion. The examiner moves the tested hip passively into extension while stabilizing unnecessary pelvic movement through palpation. The hip extension ROM was measured just before the start of pelvic anteversion; (c) the flexibility of the rectus femoris in the prone position, as well as the iliopsoas ${ }^{23}$ and hamstrings muscles in supine position was also measured.

Regarding the measurement of muscle strength, the parameters measured included the (1) spine and (2) lower limb. The parameters related to (1) spine are: (a) time loaded standing (TLS): While holding a $1 \mathrm{~kg}$ dumbbell in both hands with the shoulder at 90 degrees flexion and elbow fully extended, the maximum time (seconds) that the participant was able to hold this position was recorded ${ }^{24)}$; (b) isometric back extension: This was measured using a Biodex isokinetic dynamometer (Biodex system 4.0, Biodex Medical Systems, NY, USA). Before starting data collection, calibration was performed accord- 
ing to the manufacturer's protocol. A spinal attachment was used and the measurement was performed three times according to the manufacturer's protocol $\left.{ }^{25}\right)$. The body weight ratio $(\% \mathrm{BW})$ of the peak torque $(\mathrm{Nm})$ was calculated and recorded. The parameters related to (2) lower limb are hip and knee extensors and flexor muscle strength which were measured according to standard manual muscle testing procedures using a handheld dynamometer (MicroFet, Hoggan, Salt Lake City, UT, USA). The bodyweight ratio $(\% \mathrm{BW})$ of the peak torque (Nm; multiplying the maximum isometric strength value by the distance from the measurement position to the joint center) was calculated.

Regarding measurement of QOL, the Roland-Morris Disability Questionnaire (RDQ), the Oswestry Disability Index (ODI), the Japanese Orthopedic Association Back Pain Questionnaire (JOABPEQ) without the social life disturbance and mental health problem components, were used.

Normality of the distribution for all dependent variables was assessed using the Shapiro-Wilk test. For analysis of the relationship between sagittal alignment and ROM/muscle strength, the Pearsons' product moment correlation coefficient was used for the normal distribution and the Spearman's rank correlation coefficient was used for the non-normal distribution. For analysis of the relationship between ROM/muscle strength and QOL the Spearman's rank correlation coefficient was used.

All analyses were performed using SPSS v21.0 (IBM Corp, Armonk, NY, USA). The level of statistical significance was set at 0.05 .

\section{RESULTS}

A total number of 29 participants were referred to the study. However, three of the participants did not meet the inclusion criteria and were excluded from the study, resulting in a total of 26 participants. From the 26 analyzed, some of the participants did not complete all the required measurements i.e. for ROM: hip extension $(n=9)$, lumbar spine $(n=20)$, thoracic spine $(n=9)$, PPUT $(n=9)$, and OWD $(n=24)$; for muscle strength: hip flexors $(n=25)$, hip extensors $(n=25)$, knee flexors $(n=25)$, knee extensors $(n=24)$, TLS $(n=25)$, and isometric back extensors $(n=9)$; for QOL: JOABPEQ $(n=9)$. The participants' characteristics and mean (SD) values for all dependent variables are given in Table 1. The correlations between each of the dependent variables are shown in Tables 2 and 3.

For the correlations between sagittal alignment and ROM/muscle strength, the results are shown in Table 2. There were significant correlations between SVA and hip extension ROM $(r=-0.73, p=0.026)$; TLK and OWD $(r=-0.51, p=0.011)$; PT and TLS $(\mathrm{r}=-0.48, \mathrm{p}=0.016)$; and PI $-\mathrm{LL}$ and TLS $(\mathrm{r}=-0.40, \mathrm{p}=0.046)$. With regards to the significant correlation between SVA and hip extension ROM, the result could be biased by the large number of dropouts $(n=9)$. All other correlations were not significant.

For the correlations between QOL and ROM/muscle strength, the results are shown in Table 3. There were significant correlations between JOABPEQ (pain related disorders) and lumbar spine total ROM ( $\mathrm{r}=-0.83, \mathrm{p}=0.039$ ); JOABPEQ (gait disturbance) and knee extension ROM ( $\mathrm{r}=0.83, \mathrm{p}=0.006)$; JOABPEQ (gait disturbance) and Iliopsoas flexibility $(\mathrm{r}=0.77$, $\mathrm{p}=0.016$ ). These significant correlations related to JOABPEQ could be biased by the large number of dropouts $(\mathrm{n}=9)$. All other correlations were not significant.

\section{DISCUSSION}

For 5 of the parameters (hip extension ROM, thoracic spine ROM, PPUT, isometric back extensor strength, and JOABPEQ) the number of participants was reduced to 9. An important reason for the high drop out included the inability to perform some of these tests due to pain or discomfort. In addition, the large amount of measurements required more than one day to complete, and participants dropped out of the study due to an unwillingness or inability to continue with follow-up assessments. However, only 2 of them had significant results (hip extension ROM and JOABPEQ) and the risk of bias in these were acknowledged in the results section. Future studies should take this into account by limiting the total number of tests and simplifying the procedure so that the measurements can be completed in one session.

Sagittal alignment is important for maintaining the center of gravity over the base of support. However, the pelvis and lower limb can compensate for any changes in sagittal alignment if the center of gravity is not too far forwards. In our study, we found that there was a significant negative relationship between SVA and hip extension ROM ( $r=-0.73, p=0.03)$. Similar results were also reported by Balzil et al ${ }^{13}$. Because SVA is a measure of upper trunk forward displacement from the center position, patients can compensate for this by pelvic retroversion to decrease the $\mathrm{SVA}^{19}$ ) if the hip extension $\mathrm{ROM}^{\text {is normal }}{ }^{26)}$. However, in our study, the increase in SVA resulted in a decreased extension ROM in the hip joint. Therefore, in this type of patients, it is possible for them to develop hip flexion contractures. It is not clear if the increase in SVA is the initial result of hip flexion contractures or decreased in hip extension ROM. Further studies should investigate this relationship between SVA, hip flexion contractures and hip extension ROM.

With regards to OWD, we instructed the participants to stand against the wall and to push back towards the wall with the head in horizontal position (without head extension). To move the head backwards, compensatory movement should occur at the thoracic (TK), thoracolumbar (TLK) and lumbar (LL) segments. However, with their back against the wall, movement at lumbar segment (LL) is obstructed by the wall, and thoracic movement (TK) is limited by the rib cage. In contrast, the TLK segments (T10 to L2) are more mobile due to their anatomical characteristics (i.e. T10 to T12 have floating ribs). Any compensation required by moving the head back during OWD will occur more easily at the TLK segment. Therefore, only 
Table 1. Participants' characteristics, and mean (SD) of sagittal alignment, ROM, muscle strength, and QOL

\begin{tabular}{|c|c|c|}
\hline & $\mathrm{n}$ & Mean \pm SD \\
\hline Age (years) & 26 & $70.9 \pm 7.5$ \\
\hline Height (cm) & 26 & $158.0 \pm 7.7$ \\
\hline Body weight (kg) & 26 & $57.3 \pm 10.2$ \\
\hline \multicolumn{3}{|l|}{ Sagittal alignment } \\
\hline Thoracic kyphosis (degrees) & 26 & $23.4 \pm 11.5$ \\
\hline Thoracolumbar kyphosis (degrees) & 26 & $13.5 \pm 12.5$ \\
\hline Lumbar lordosis (degrees) & 26 & $19.4 \pm 18.7$ \\
\hline Sagittal vertical axis (mm) & 26 & $89.5 \pm 42.6$ \\
\hline Pelvic incidence (degrees) & 26 & $49.4 \pm 13.1$ \\
\hline Pelvic tilt (degrees) & 26 & $31.1 \pm 10.3$ \\
\hline Sacral slope (degrees) & 26 & $18.2 \pm 13.1$ \\
\hline PI - LL (degrees) & 26 & $30.0 \pm 15.5$ \\
\hline \multicolumn{3}{|l|}{ Range of motion } \\
\hline Hip joint flexion (degrees) & 26 & $110.4 \pm 6.5$ \\
\hline Hip joint extension (degrees) & 9 & $7.9 \pm 5.8$ \\
\hline Knee joint flexion (degrees) & 26 & $143.0 \pm 7.4$ \\
\hline Knee joint extension (degrees) & 26 & $-4.0 \pm 3.6$ \\
\hline Lumbar spine (degrees) & 20 & $25.3 \pm 10.3$ \\
\hline Thoracic spine (degrees) & 9 & $44.7 \pm 13.9$ \\
\hline Prone press-up test $(\mathrm{cm})$ & 9 & $19.7 \pm 5.8$ \\
\hline Occiput to wall distance $(\mathrm{cm})$ & 24 & $4.1 \pm 4.1$ \\
\hline Rectus femoris flexibility (degrees) & 26 & $129.7 \pm 10.3$ \\
\hline Hamstrings flexibility (degrees) & 26 & $60.7 \pm 10.3$ \\
\hline Iliopsoas flexibility (degrees) & 26 & $15.3 \pm 7.9$ \\
\hline \multicolumn{3}{|l|}{ Muscle strength } \\
\hline Hip joint flexion (\% BW) & 25 & $0.9 \pm 0.3$ \\
\hline Hip joint extension (\% BW) & 25 & $0.9 \pm 0.3$ \\
\hline Knee joint flexion (\% BW) & 25 & $0.5 \pm 0.2$ \\
\hline Knee joint extension (\% BW) & 24 & $0.8 \pm 0.2$ \\
\hline Time loaded standing (seconds) & 25 & $48.3 \pm 39.9$ \\
\hline Isometric back extensors (\% BW) & 9 & $232.5 \pm 98.1$ \\
\hline \multicolumn{3}{|l|}{ Quality of life } \\
\hline Roland-Morris Disability Questionnaire & 26 & $7.0 \pm 4.5$ \\
\hline Oswestry Disability Index & 26 & $29.4 \pm 14.7$ \\
\hline \multicolumn{3}{|l|}{ JOABPEQ } \\
\hline Pain-related disorders & 9 & $53.8 \pm 31.7$ \\
\hline Lumbar dysfunction & 9 & $67.7 \pm 20.6$ \\
\hline Gait disturbance & 9 & $57.0 \pm 24.7$ \\
\hline
\end{tabular}

\% BW: percentage body weight; PI - LL: Pelvic incidence minus lumbar lordosis; JOABPEQ: Japanese Orthopedic Association Back Pain Questionnaire.

movement at the thoracolumbar (TLK) segment during OWD was possible and a significant positive correlation was found between OWD and TLK ( $\mathrm{r}=0.51, \mathrm{p}=0.01$ ), while the rest (TK and LL) was not significantly correlated with OWD.

Our results also demonstrated a significant relationship between pelvic tilt (PT) and time-loaded standing (TLS). TLS is a measure of spinal extensor muscle endurance. In participants with kyphosis, there is an increased flexor moment in the spine due to the center of gravity of the trunk being too forward. To compensate for this, participants usually will tilt their pelvis backwards (retroversion) resulting in increased PT. Therefore, if TLS is poor, PT (retroversion) will be increased, i.e. a negative correlation between TLS and PT as shown in our results $(\mathrm{r}=-0.48, \mathrm{p}=0.02)$.

Lumbar lordosis is usually measured by LL angle. However, according to Barrey et al. ${ }^{19)}$ the more accurate measure of lumbar lordosis should consider the position of the hip joint relative to the sacrum (PI). Therefore, Schwab et al..$^{20)}$ suggested that rather than LL, the PI - LL mismatch is more important because it is a better indicator of severity for adult spinal 
Table 2. Pearson's correlation coefficients for sagittal alignment, ROM and muscle strength

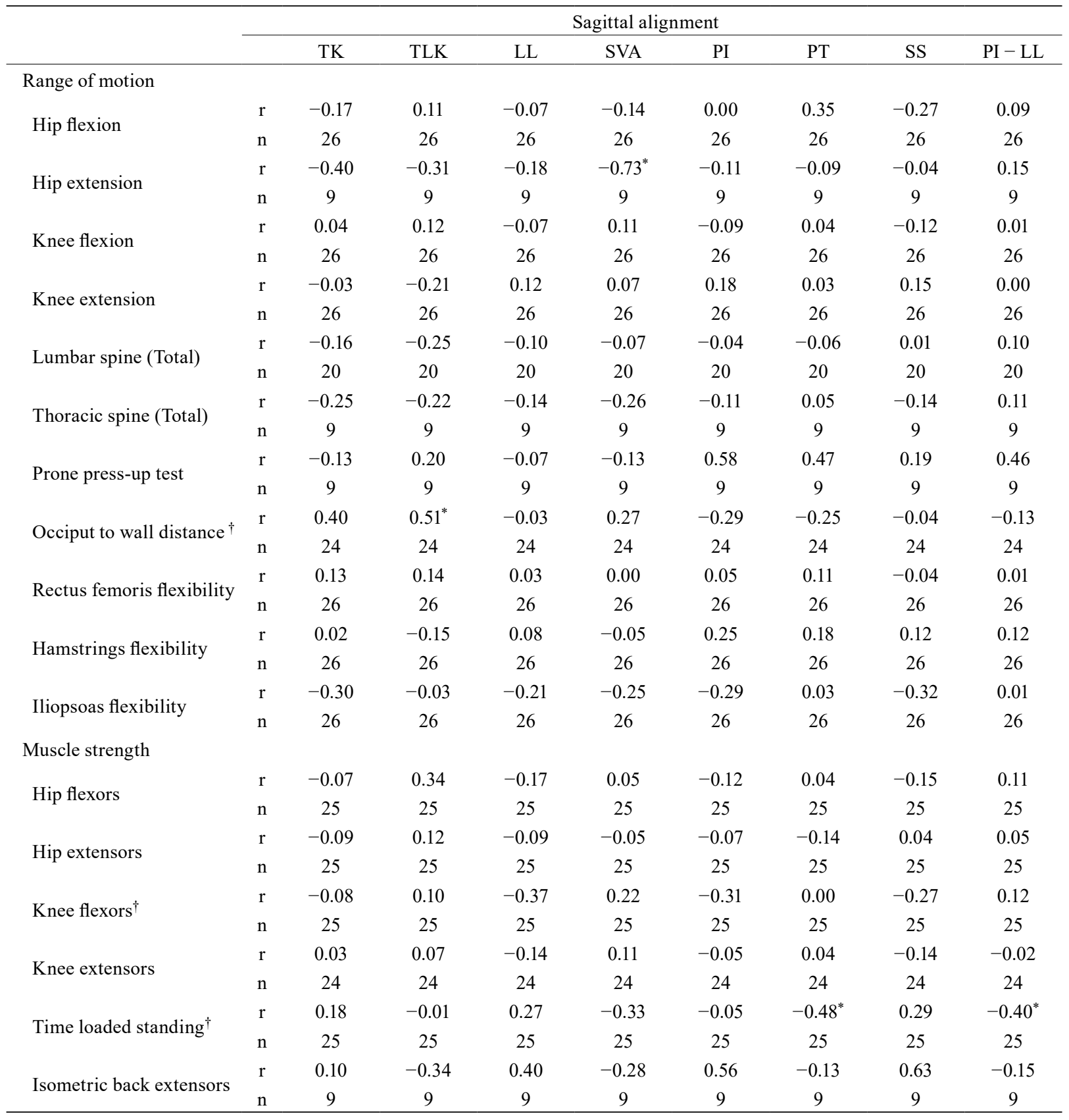

TK: Thoracic kyphosis; TLK: Thoracolumbar kyphosis; LL: Lumbar lordosis; SVA: Sagittal vertical axis; PI: Pelvic incidence; PT: pelvic tilt; SS: Sacral slope; PI - LL; Pelvic incidence minus lumbar lordosis; ${ }^{\dagger}$ Spearman correlation coefficient; ${ }^{*} \mathrm{p}<0.05$.

$\mathrm{n}$ values ranged from 9 to 26 due to dropout. Reasons for the drop out included the inability to perform some of these tests due to pain or discomfort. In addition, the large amount of measurements required more than one day to complete, and participants dropped out of the study due to an unwillingness or inability to continue with follow-up assessments.

deformity. If the PI - LL mismatch is small, the patient is able to compensate by pelvic retroversion ${ }^{19}$. In our study, we found a negative significant correlation between PI - LL and TLS ( $r=-0.40, p=0.046)$. This can be attributed to decreased flexor moment in the spine resulting in an increased in the PI - LL mismatch. In contrast, isometric back extensor strength did not correlate with any of the alignment parameters. Since our study showed that TLS was correlated with sagittal alignment (PT and PI - LL), this suggests that endurance (i.e. TLS) rather than isometric back extensor muscle strength is a better measure for clinical evaluation. Traditionally, exercise therapy for patients with kyphosis emphasized increasing spinal extensor muscle strength. Our study suggests that increasing spinal extensor muscle endurance may be more important than muscle strength.

Normal ROM and muscle strength may be important factors for achieving a high QOL ${ }^{27,28)}$. In our study, JOABPEQ (pain related disorders) was correlated with ROM in the lumbar spine ( $r=-0.83, \mathrm{p}=0.039)$. The higher the score for JOABPEQ (pain 
Table 3. Spearman's correlation coefficients for QOL and ROM and muscle strength

\begin{tabular}{|c|c|c|c|c|c|c|}
\hline & & \multirow[b]{2}{*}{ RDQ } & \multirow[b]{2}{*}{ ODI } & \multicolumn{3}{|c|}{ JOABPEQ } \\
\hline & & & & Pain & $\begin{array}{c}\text { Lumbar } \\
\text { dysfunction }\end{array}$ & Gait \\
\hline \multicolumn{7}{|l|}{ Range of motion } \\
\hline \multirow{2}{*}{ Hip flexion } & $\mathrm{r}$ & -0.04 & 0.25 & -0.28 & -0.23 & -0.10 \\
\hline & $\mathrm{n}$ & 26 & 26 & 9 & 9 & 9 \\
\hline \multirow{2}{*}{ Hip extension } & $\mathrm{r}$ & -0.09 & 0.23 & -0.05 & 0.17 & 0.24 \\
\hline & $\mathrm{n}$ & 9 & 9 & 9 & 9 & 9 \\
\hline \multirow{2}{*}{ Knee flexion } & $\mathrm{r}$ & -0.07 & 0.20 & -0.16 & 0.31 & -0.28 \\
\hline & $\mathrm{n}$ & 26 & 26 & 9 & 9 & 9 \\
\hline \multirow{2}{*}{ Knee extension } & $\mathrm{r}$ & 0.08 & -0.01 & -0.57 & -0.22 & $0.83^{* *}$ \\
\hline & $\mathrm{n}$ & 26 & 26 & 9 & 9 & 9 \\
\hline \multirow{2}{*}{ Lumbar spine (total) } & $\mathrm{r}$ & 0.04 & 0.06 & $-0.83^{*}$ & 0.32 & 0.67 \\
\hline & $\mathrm{n}$ & 20 & 20 & 6 & 6 & 6 \\
\hline \multirow{2}{*}{ Thoracic spine (total) } & $\mathrm{r}$ & -0.60 & 0.28 & 0.05 & 0.40 & -0.03 \\
\hline & $\mathrm{n}$ & 9 & 9 & 9 & 9 & 9 \\
\hline \multirow{2}{*}{ Prone press-up test } & $\mathrm{r}$ & 0.07 & 0.13 & 0.40 & -0.15 & 0.17 \\
\hline & $\mathrm{n}$ & 9 & 9 & 9 & 9 & 9 \\
\hline \multirow{2}{*}{ Occiput to wall distance } & $\mathrm{r}$ & 0.11 & 0.06 & 0.38 & -0.08 & -0.28 \\
\hline & $\mathrm{n}$ & 24 & 24 & 8 & 8 & 8 \\
\hline \multirow{2}{*}{ Rectus femoris flexibility } & $\mathrm{r}$ & 0.07 & 0.14 & -0.15 & -0.02 & -0.32 \\
\hline & $\mathrm{n}$ & 26 & 26 & 9 & 9 & 9 \\
\hline \multirow{2}{*}{ Hamstrings flexibility } & $\mathrm{r}$ & -0.04 & 0.01 & 0.17 & -0.21 & -0.24 \\
\hline & $\mathrm{n}$ & 26 & 26 & 9 & 9 & 9 \\
\hline \multirow{2}{*}{ Iliopsoas flexibility } & $\mathrm{r}$ & 0.34 & 0.26 & -0.56 & -0.09 & $0.77 *$ \\
\hline & $\mathrm{n}$ & 26 & 26 & 9 & 9 & 9 \\
\hline \multicolumn{7}{|l|}{ Muscle strength } \\
\hline \multirow{2}{*}{ Hip flexors } & $\mathrm{r}$ & -0.12 & 0.10 & 0.33 & -0.19 & -0.20 \\
\hline & $\mathrm{n}$ & 25 & 25 & 9 & 9 & 9 \\
\hline \multirow{2}{*}{ Hip extensors } & $\mathrm{r}$ & 0.25 & 0.20 & -0.01 & -0.12 & 0.29 \\
\hline & $\mathrm{n}$ & 25 & 25 & 9 & 9 & 9 \\
\hline \multirow{2}{*}{ Knee flexors } & $\mathrm{r}$ & 0.13 & 0.16 & -0.17 & -0.30 & 0.41 \\
\hline & $\mathrm{n}$ & 25 & 25 & 9 & 9 & 9 \\
\hline \multirow{2}{*}{ Knee extensors } & $\mathrm{r}$ & 0.19 & 0.31 & 0.66 & -0.41 & -0.19 \\
\hline & $\mathrm{n}$ & 24 & 24 & 8 & 8 & 8 \\
\hline \multirow{2}{*}{ Time loaded standing } & $\mathrm{r}$ & -0.39 & -0.28 & 0.50 & -0.11 & -0.39 \\
\hline & $\mathrm{n}$ & 25 & 25 & 8 & 8 & 8 \\
\hline \multirow{2}{*}{ Isometric back extensors } & $\mathrm{r}$ & -0.24 & -0.55 & 0.41 & -0.35 & 0.10 \\
\hline & $\mathrm{n}$ & 9 & 9 & 9 & 9 & 9 \\
\hline
\end{tabular}

RDQ: Roland-Morris Disability Questionnaire; ODI: Oswestry Disability Index; JOABPEQ: Japanese Orthopaedic Association Back Pain Questionnaire; ${ }^{*} \mathrm{p}<0.01 ;{ }^{*} \mathrm{p}<0.05$.

$\mathrm{n}$ values ranged from 6 to 26 due to dropout. Reasons for the drop out included the inability to perform some of these tests due to pain or discomfort. In addition, the large amount of measurements required more than one day to complete, and participants dropped out of the study due to an unwillingness or inability to continue with follow-up assessments.

related disorders), the lower the pain. In other words, as the lumbar spine ROM decreases, pain decreases or vice-versa. In addition, JOABPEQ (gait disturbance) were correlated with knee extension ROM ( $\mathrm{r}=0.83, \mathrm{p}=0.006)$ and Iliopsoas flexibility $(\mathrm{r}=0.77, \mathrm{p}=0.016)$. The higher the score for JOABPEQ (gait disturbance) the lower the gait disturbance. In other words, as ROM decreases, gait disturbance increases. So, any limitations in knee extension ROM and iliopsoas flexibility would result in increased gait disturbance. However, it can also be due to unknown causes and may require further investigation.

A limitation of our study was the high dropout rate especially for ROM and isometric back extensor muscle strength on the Biodex isokinetic dynamometer. All measurements were done over two days across one week and ROM and back extensor muscle strength were scheduled on the second day. Future studies should try to limit all measurements to one day, as well as increase the sample size in order to account for unexpected drop out rate. 
In conclusion, our study demonstrated that hip extension ROM and back extensor endurance were important factors in sagittal alignment, and lumbar spine ROM, as well as hip and knee extension ROM were important factors for QOL. These factors should be targeted for assessment and treatment in adults with spinal deformities.

\section{Funding and Conflict of interest}

None.

\section{REFERENCES}

1) Yamato Y, Matsuyama Y, Ito M, et al.: Radiographic parameter of adult spinal deformity. Seikei Geka, 2013, 64: 841-844 (in Japanese).

2) Fon GT, Pitt MJ, Thies AC Jr: Thoracic kyphosis: range in normal subjects. AJR Am J Roentgenol, 1980, 134: 979-983. [Medline] [CrossRef]

3) Hinman MR: Comparison of thoracic kyphosis and postural stiffness in younger and older women. Spine J, 2004, 4: 413-417. [Medline] [CrossRef]

4) Kanemura T, Imagama S: Spino-pelvic alignment in an asymptomatic Japanese population — mean and variance of upright thoracolumbar spino-pelvic alignment in Japanese. Kansetsugeka, 2009, 28: 10-19 (in Japanese).

5) Kobayashi T, Atsuta Y, Ito H, et al.: Evaluation of spinal deformity among elderly and conservative treatment. Seikei Geka, 2013, 64: 857-861 (in Japanese).

6) Tran TH, Wing D, Davis A, et al.: Correlations among four measures of thoracic kyphosis in older adults. Osteoporos Int, 2016, 27: 1255-1259. [Medline] [CrossRef]

7) Öhlén G, Spangfort E, Tingvall C: Measurement of spinal sagittal configuration and mobility with Debrunner's kyphometer. Spine, 1989, 14: 580-583. [Medline] [CrossRef]

8) Azadinia F, Kamyab M, Behtash H, et al.: The validity and reliability of noninvasive methods for measuring kyphosis. J Spinal Disord Tech, 2014, 27: E212E218. [Medline] [CrossRef]

9) Mannion AF, Knecht K, Balaban G, et al.: A new skin-surface device for measuring the curvature and global and segmental ranges of motion of the spine: reliability of measurements and comparison with data reviewed from the literature. Eur Spine J, 2004, 13: 122-136. [Medline] [CrossRef]

10) Katzman WB, Wanek L, Shepherd JA, et al.: Age-related hyperkyphosis: its causes, consequences, and management. J Orthop Sports Phys Ther, 2010, 40: 352-360. [Medline] [CrossRef]

11) Glassman SD, Bridwell K, Dimar JR, et al.: The impact of positive sagittal balance in adult spinal deformity. Spine, 2005, 30: 2024-2029. [Medline] [CrossRef]

12) Sinaki M, Itoi E, Rogers JW, et al.: Correlation of back extensor strength with thoracic kyphosis and lumbar lordosis in estrogen-deficient women. Am J Phys Med Rehabil, 1996, 75: 370-374. [Medline] [CrossRef]

13) Balzini L, Vannucchi L, Benvenuti F, et al.: Clinical characteristics of flexed posture in elderly women. J Am Geriatr Soc, 2003, 51: 1419-1426. [Medline] [CrossRef]

14) Kai Y, Murata S, Otao H, et al.: A relationship between spinal deformity and lower limb muscle strength in community-dwelling elderly females. Rigakuryohokagaku, 2009, 24: 45-48 (in Japanese). [CrossRef]

15) Moal B, Bronsard N, Raya JG, et al.: Volume and fat infiltration of spino-pelvic musculature in adults with spinal deformity. World J Orthop, 2015, 6: 727-737. [Medline] [CrossRef]

16) Bansal S, Katzman WB, Giangregorio LM: Exercise for improving age-related hyperkyphotic posture: a systematic review. Arch Phys Med Rehabil, 2014, 95: 129-140. [Medline] [CrossRef]

17) Saimon $Y$, Ryuzaki D, Akahane H, et al.: Correlation between sagittal alignment, range of motion, muscle strength of the spine and lower limbs and quality of life in female adults with spinal deformities — a pilot study—. Rigakuryouhoukenkyuu Nagano, 2017, 46: 16-19.

18) Horton WC, Brown CW, Bridwell KH, et al.: Is there an optimal patient stance for obtaining a lateral 36 " radiograph? A critical comparison of three techniques. Spine, 2005, 30: 427-433. [Medline] [CrossRef]

19) Barrey C, Roussouly P, Le Huec JC, et al.: Compensatory mechanisms contributing to keep the sagittal balance of the spine. Eur Spine J, 2013, 22: S834-S841. [Medline] [CrossRef]

20) Schwab F, Ungar B, Blondel B, et al.: Scoliosis Research Society-Schwab adult spinal deformity classification: a validation study. Spine, 2012, 37: 1077-1082. [Medline] [CrossRef]

21) Bandy WD, Reese NB: Strapped versus unstrapped technique of the prone press-up for measurement of lumbar extension using a tape measure: differences in magnitude and reliability of measurements. Arch Phys Med Rehabil, 2004, 85: 99-103. [Medline] [CrossRef]

22) Pourahmadi MR, Taghipour M, Jannati E, et al.: Reliability and validity of an iPhone ${ }^{\circledR}$ application for the measurement of lumbar spine flexion and extension range of motion. PeerJ, 2016, 4: e2355. [Medline] [CrossRef]

23) Pua YH, Wrigley TV, Cowan SM, et al.: Intrarater test-retest reliability of hip range of motion and hip muscle strength measurements in persons with hip osteoarthritis. Arch Phys Med Rehabil, 2008, 89: 1146-1154. [Medline] [CrossRef]

24) Shipp KM, Purse JL, Gold DT, et al.: Timed loaded standing: a measure of combined trunk and arm endurance suitable for people with vertebral osteoporosis Osteoporos Int, 2000, 11: 914-922. [Medline] [CrossRef]

25) Katzman WB, Sellmeyer DE, Stewart AL, et al.: Changes in flexed posture, musculoskeletal impairments, and physical performance after group exercise in community-dwelling older women. Arch Phys Med Rehabil, 2007, 88: 192-199. [Medline] [CrossRef]

26) Roussouly P, Pinheiro-Franco JL: Biomechanical analysis of the spino-pelvic organization and adaptation in pathology. Eur Spine J, 2011, 20: 609-618. [Medline] [CrossRef]

27) Gonçalves LC, Vale RG, Barata NJ, et al.: Flexibility, functional autonomy and quality of life (QoL) in elderly yoga practitioners. Arch Gerontol Geriatr, 2011, 53: 158-162. [Medline] [CrossRef]

28) Imagama S, Matsuyama $Y$, Hasegawa $Y$, et al.: Back muscle strength and spinal mobility are predictors of quality of life in middle-aged and elderly males. Eur Spine J, 2011, 20: 954-961. [Medline] [CrossRef] 